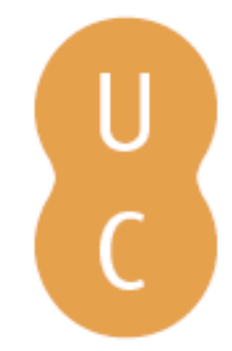

\title{
nommalina
}

\section{Utopía, espacios soñados y mito clásico en la Tragicomedia de Los Jardines y Los} Campos Sabeos de Feliciana Enríquez de Guzmán
Autor(es): $\quad$ Rosa Cubo, Cristina de la
Publicado por: Associação Portuguesa de Estudos Clássicos; Imprensa da
URL persistente: Universidade de Coimbra
DOI:
URI:http://hdl.handle.net/10316.2/31816
DOI:http://dx.doi.org/10.14195/978-972-98142-2-8_9
Accessed : $\quad$ 26-Apr-2023 10:32:57

A navegação consulta e descarregamento dos títulos inseridos nas Bibliotecas Digitais UC Digitalis, UC Pombalina e UC Impactum, pressupõem a aceitação plena e sem reservas dos Termos e Condições de Uso destas Bibliotecas Digitais, disponíveis em https://digitalis.uc.pt/pt-pt/termos.

Conforme exposto nos referidos Termos e Condições de Uso, o descarregamento de títulos de acesso restrito requer uma licença válida de autorização devendo o utilizador aceder ao(s) documento(s) a partir de um endereço de IP da instituição detentora da supramencionada licença.

Ao utilizador é apenas permitido o descarregamento para uso pessoal, pelo que o emprego do(s) título(s) descarregado(s) para outro fim, designadamente comercial, carece de autorização do respetivo autor ou editor da obra.

Na medida em que todas as obras da UC Digitalis se encontram protegidas pelo Código do Direito de Autor e Direitos Conexos e demais legislação aplicável, toda a cópia, parcial ou total, deste documento, nos casos em que é legalmente admitida, deverá conter ou fazer-se acompanhar por este aviso.

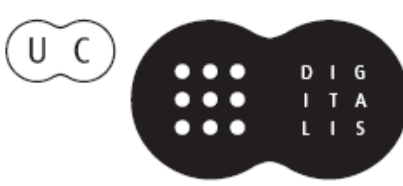




\section{Espaços e Paisagens}

\section{Antiguidade Clássica e Heranças Contemporâneas}

Vol. Il Línguas e Literaturas. Idade Média. Renascimento. Recepção

Francisco de Oliveira, Cláudia Teixeira, Paula Barata Dias (Coords.)

IMPRENSA DA UNIVERSIDADE DE COIMBRA 


\title{
UTOPÍA, ESPACIOS SOÑADOS Y MITO CLÁSICO EN LA TRAGICOMEDIA DE LOS JARDINES Y LOS CAMPOS SABEOS DE FELICIANA ENRÍQUEZ DE GUZMÁN
}

\author{
Cristina de la Rosa Cubo \\ Universidad de Valladolid
}

\begin{abstract}
The Spanish playwright, Feliciana Enríquez de Guzmán, devoted many years of her life to the composition of a tragicomedy where she defines herself as a defender of the precepts of Greco.Latin theatre. The play takes place in a legendary time where men coexist with the ancient Gods and is used by the author as a reflection of a dream reality, which provides answers to her worries and releases her true ambitions and vital expectations. In the play, the author presents the understanding father and own free will which she never had and the transgression capacity that the society always denied. Thus, the tragicomic world which so well describes the society, the culture and the politics of her days, turns frequently into a distorted image of the daily atmosphere in the Spanish XVII century, by offering an irreverent and amusing view of the classical myth.
\end{abstract}

Keywords: classical myth, Feliciana Enríquez de Guzmán, garden, locus amoenus, transgression, woman.

Palavras-chave: Feliciana Enríquez de Guzmán, jardim, locus amoenus, mito clássico, mulher, transgressão.

Feliciana Enríquez de Guzmán ${ }^{1}$ termina su obra "la Tragicomedia de los Jardines y los Campos Sabeos" en $1519^{2}$. El lugar elegido por Feliciana para enmarcar la ficción literaria de su única obra es un jardín de gran valor simbólico como veremos. El jardín en el teatro barroco se ha estudiado como elemento esencial del espacio escénico en el teatro cortesano ${ }^{3}$, un espacio en el que se desarrolla la puesta en escena de la obra teatral, que sigue las convenciones de la época y que tiene una innegable relación con el texto dramático ${ }^{4}$.

${ }^{1}$ En anteriores ocasiones he abordado el estudio del mundo clásico en la obra de Feliciana: C. de la Rosa Cubo (2005). P. Conde Parrado, C. de la Rosa (2005).

${ }^{2}$ Feliciana Enríquez de Guzmán escribió bajo el título de La Tragicomedia de los Jardines y los Campos Sabeos dos tragicomedias en verso de cinco actos cada una con coros y entreactos. Una fiesta teatral de la que no ha existido una edición moderna y completa hasta la aparición de la obra de Louis Pérez en La tragicomedia de los jardines y los Campos Sabeos: L. Pérez 1988. Es la edición que seguimos en nuestras citas.

${ }^{3}$ T. Ferrer Valls 1991.

${ }^{4}$ O. Arróniz 1977. J. M. Díez Borque 1991. A. Egido 1989. J. De Lara Garrido, 1995. 
Por supuesto, también debemos relacionar las continuas alusiones al motivo del jardín con el tópico del locus a moenus ${ }^{5}$, acuñado por la antigüedad grecolatina y presente en todas las épocas literarias. Recordemos que el locus amoenus parte del escenario de la poesía bucólica de tema amoroso e idílico. Como señaló Robert Curtius ${ }^{6}$, el paisaje ameno suele penetrar asimismo en la descripción poética de los jardines: "El locus amoenus es un paraje hermoso y umbrío, sus elementos esenciales son un árbol (o varios), un prado y una fuente o arroyo; a ellos pueden añadirse un canto de aves, unas flores y, aún más, el soplo de la

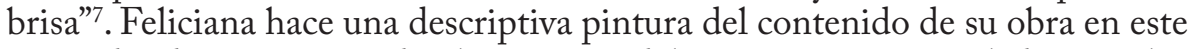
texto, donde aparecen todos los tópicos del locus amoenus mezclados con los elementos característicos del amor caballeresco:

Jardines, huertas, campos, bosques, ríos;

Sueños, máscaras, letras, cartas, joyas;

Afectos amorosos, castos, puros;

Flores, donayres, danças, bailes, músicas;

Torneos, luchas, coros desposorios (...)(Pérez 45)

Unos jardines que se alejan del tópico del hortus conclusus, pues una de sus características, que se describe en los primeros versos del acto primero, es la ausencia de muros y puertas, en clara alusión a la libertad de los personajes que en ellos moran:

\author{
De un agravio acerbo, $y$ duro \\ estos jardines se quexan: \\ que sin defensa los dexan \\ de cerca, torres y muro. \\ campos, valles, bosques, prados \\ rio, jardines y huertas \\ todo está junto sin puertas \\ sin paredes ni vallados. (Pérez 46)
}

La inmovilidad y perfección vegetal remite a un espacio único donde el tiempo no transcurre. Los jardines descritos están fuera del tiempo real, como si se tratase de un jardín ficticio que se viese en una esfera de cristal, pues, por definición, los jardines se oponen a la naturaleza por el hecho de detener el tiempo con una vegetación siempre viva, a la que no afecta el paso de las estaciones porque su vegetación posee una frescura perenne ${ }^{8}$ Feliciana Enríquez, manteniendo la unidad de lugar del teatro clásico, sitúa los 5 actos de cada una de las partes de su tragicomedia en los míticos Jardines de Saba, así lo afirma en el prólogo?:

${ }^{5}$ C. Hernández Varcárcel 1984.

${ }^{6}$ Cf E. R.Curtius 1977: 280-282

${ }^{7}$ Ibid. p. 280

${ }^{8}$ A. Egido 1990: 116.

${ }^{9}$ Feliciana sigue las leyes dramáticas del Arte clásico grecolatino, manteniendo la estructura 378 


\title{
Si estos preceptos con rigor guardados, \\ Yo, señores os diesse dos comedias (...) \\ $Y$ en todas ellas siempre un mismo sitio, \\ Siempre un mismo lugar, en los Jardines, \\ En la primera parte en toda ella. \\ Y en la segunda por la propia forma \\ En los campos Sabéos observasse? (Pérez 45)
}

Si es común como hemos dicho, la aparición del jardín en el teatro barroco, no lo es tanto que se precise su situación geográfica, la fértil región de Saba, en lo que, por aquel entonces, se conoce como la Arabia Felix. Tanto Saba ${ }^{10}$ como el adjetivo sabeo es referencia corriente en la literatura de la época ${ }^{11}$, evoca un lugar lejano, y hace referencia a su mítica reina y al incienso que allí se produce. Feliciana no explica la razón que le lleva a localizar su obra en este espacio concreto, una explicación podría ser la semejanza fonética de Sevilla-Saba, pues ella declara en uno de sus prólogos que los hechos narrados en Arabia, han sucedido realmente en Sevilla:

\author{
De dos amantes que en sus tiernos años \\ Se amaron y adoraron con invidia, \\ $Y$ emulación de muchos enemigos, \\ Desde el primero instante, en que se vieron, \\ $Y$ en el mismo en sus almas dulcemente \\ Con reciproco amor se transformaron; \\ Aunque ella se mudó, y a el que fue firme, \\ Remuneró el muy Alto con ventajas; \\ La bistoria en nuestros tiempos sucedida, \\ Que vio el famoso Betis y otro río, \\ $Y$ hoy leen escrita por sus verdes álamos; \\ Cifra nuestra Poeta Sevillana \\ En su Tragicomedia, que en Arabia, \\ Finge haber sucedido en los Sabeos, \\ Campos, y sus jardines, que gozaron \\ Los Amores de Venus, y su Adonis. ( Pérez 42-43)
}

Claramente vemos en las palabras de Feliciana que sus intenciones son dejar constancia de sucesos autobiográficos amparada en la farsa teatral, y, efectivamente, en la Tragicomedia el hilo conductor son los amores humanos y divinos, el jardín desaparece como motivo literario, pierde protagonismo y deja

de cinco actos y las unidades de acción, tiempo y espacio

${ }^{10}$ En uno de los entreactos, Feliciana lo utiliza de forma paródica como nombre de uno de los pretendientes de las gracias mohosas.

11 "Pero no me negarás, Sancho, una cosa: cuando llegaste junto a ella, ¿̇no sentiste un olor sabeo, una fragancia aromática, y un no sé qué de bueno, que yo no acierto a dalle nombre?" Capítulo xxxi (Primera parte del Quijote). 
paso al sentimiento amoroso que lo invade todo. Amores humanos que siguen el código del amor cortés y esconden los sentimientos de la autora y amores divinos que consiguen la fusión del mundo mítico con el caballeresco y en los que la autora se permite toda una serie de transgresiones morales ${ }^{12}$ ya presentes en la mitología clásica- que rompen el "amor honesto y puro" que se profesan los protagonistas mortales.

Una joven en la Sevilla de comienzos del siglo XVII, tenía como destino el matrimonio impuesto por sus padres, Feliciana Enríquez fue obligada a casarse con el viudo Don Cristóbal Ponce Solís, pero cuando comenzó a escribir esta historia sólo podía temer que se cumpliera el destino de tantas otras mujeres. Ya casada, siguió escribiendo la Tragicomedia y pudo verter sus vivencias autobiográficas en la obra. Podemos imaginar que el carácter independiente de Feliciana le hacía manifestar su protesta hacia los varones considerados como un "buen partido" por sus progenitores, su repulsa la lleva a autorepresentarse mediante personajes femeninos míticos que huyen del contacto de un hombre. Como ejemplo de la fascinación que Feliciana siente por las mujeres mitológicas que rehúsan el contacto con varones, Feliciana introduce en su obra la trágica historia de Atalante, Dafne y Siringa estudiada en otros trabajos. ${ }^{13}$

Tres años más tarde muere su marido y unos meses después Feliciana se casa con su primer amor, al que no había podido olvidar: D. Francisco de León Garabito. Los hombres de su vida tienen también una clara representación en la obra: Francisco de León es el príncipe de Esparta Clarisel, el héroe esforzado cuya lealtad le hace esperar a que Feliciana recupere su libertad, nobleza que fue premiada con las bodas entre ambos. Su primer marido Cristóbal Ponce Solís es Vulcano, viejo y poco atractivo, de quien se burlan unos Cupidillos que justifican la infidelidad de su esposa joven y bella. Venus es la esposa traidora, que obtiene la simpatía del lector, comprensivo ante sus amores con Adonis, motivados por la poca atracción que siente por un esposo repulsivo y decrépito:

\author{
Di viejo verde \\ como quieres concuerde \\ con tu pie cojo y copo, \\ con tus manos tiznadas \\ con tus piernas quebradas \\ con tu boca soplona, ojos de topo, \\ los ojos, tez, blancura, \\ la gracia de mi madre y hermosura? ( Pérez 213)
}

${ }^{12} \mathrm{La}$ actitud moral transgresora es una constante en los personajes míticos y alguno de los secundarios: poliandria, travestismo, incesto, adulterio...sin embargo los protagonistas de la Primera parte Clarisel y Belidiana y los de la segunda Clarisel y Maya, mantienen una actitud aceptable para las convenciones morales de la época. No comparto la teoría de Reina Ruiz quien ve en la relación de Belidiana y su padre una clara intención incestuosa, a mi juicio, inexistente. Cf. M. R. Ruiz 2005: 39-68

${ }^{13}$ P. Conde Parrado, C. de la Rosa, 2005: 253-265 
En una obra de claros tintes autobiográficos el tema del amor merece ser tratado en capítulo aparte. Aparecen aquí todos los sentimientos que puede despertar la pasión amorosa: desde la soledad motivada por la ausencia a la desesperación que produce la pérdida del ser amado pasando por la alegría desbordante del amor correspondido. En la obra asoman dos Felicianas: la real, que tuvo que acatar la decisión paterna y casar con un viudo a pesar de sus propios deseos, y la Feliciana soñada, una mujer independiente y audaz que aparece bajo el sobrenombre de Maya.

La Feliciana real se somete a la voluntad del padre implacable ${ }^{14}$, a pesar de que no coincidan con sus propios deseos. Y sufre, por la ausencia de su amado, atroces tormentos, expresados con sentimientos a flor de piel: la ausencia es muerte fiera, los padres crueles alimañas y ella la víctima y trofeo de esta feroz cacería ${ }^{15}$.

La soledad, durante los tres años que duró su matrimonio, es también un tema de la obra que expresa en el Coro del acto IV:

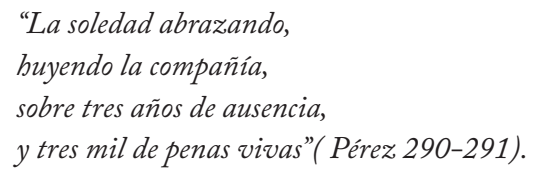

Recuerdos que hieren el alma de los amantes y hacen increpar a la Fortuna que les permitió conocerse para luego separarles y que simboliza con el mito de los amores imposibles de Eco y Narciso ${ }^{16}$.

Tristeza que se expresa en una queja hacia el amado ${ }^{17}$. Desesperación que se convierte en locura recordando el amor perdido en el Coro del acto III. Es la dramática reacción de Venus ante la muerte de Adonis, la enajenación motivada por la pérdida de la persona amada:

\section{Tuerce la diosa sus manos \\ y besa las que excluyeron \\ con su amorosa blancura \\ las tiznadas del herrero. \\ La guirnalda que ceñia \\ el oro de sus cabellos, \\ con los dientes despedaza, \\ como mordida de perro. \\ Hiere sus sacras mexillas, \\ y a las difuntas da besos;}

\footnotetext{
14 "Ay, Clarisel, pues tuve suerte negra/en padre porfiado y riguroso" (Pérez 1988 191)

15 Pérez, 164, C.

${ }^{16}$ Pérez, 288-289.

17 "Vayan a ti mis suspiros/pues eres el que los formas/la razón por que los doy,/bien sé que tú no la ignoras” (Pérez, 314-315.
} 
muerde sus labios, y pone

los del joven muerto en ellos.

Sus blancos braços enlaça

al blanco alabastro terso

de la garganta, que fue

su paz, su gloria, y consuelo.

(Pérez 216)

A pesar de la lejanía impuesta, el enamorado mantiene su lealtad por Feliciana. La lealtad en la relación amorosa se convierte en motivo recurrente de la expresión poética, es el "foedus amoris", el pacto de amor de Catulo hacia Lesbia en la poesía clásica latina, la condición indispensable para que amor llegue a buen fin y que, si se rompe, convierte en sacrílego al que lo incumple ante la mirada de los dioses.

\author{
(...) Porque primero a otro \\ fue prometida por amada esposa \\ de la muger, y potro \\ la fuerza es peligrosa, \\ que quieren mano blanda, y amorosa. \\ Mas no quiero culparte, \\ Arroyo ilustre, que antes fuera amencia. \\ De tal bien despojarte; \\ llore ella su demencia, \\ que no tuvo valor ni fe en ausencia. \\ $Y$ tu, Ojoven constante, \\ Ten ánimo en los golpes de Fortuna, \\ Que a tan leal amante, \\ Guardada tienen una \\ Los hados en la esfera de la luna. \\ Esta es la linda maya, \\ Que en la ciudad hercúlea de Fernando, \\ Por la línea Pelaya \\ Su estirpe derivando, \\ Será princesa del piérido vando. (...) (Pérez, 290)
}

La segunda Feliciana que aparece en la obra lleva el nombre de la mítica Maya, la mayor de las Pléyades a la que no se le pone nada por delante, la misma Venus es su aliada y favorece sus deseos. Maya se reencuentra con Francisco de León en la segunda parte de la Tragicomedia (lo mismo que en la vida real) y ya nadie puede separarles: la relación se convierte en una gozosa descripción de amor y de deseo en el que resuenan los ecos del Cantar de los cantares de 
Fray Luis de León ${ }^{18}$, y se legitima mediante un solemne matrimonio en el que no faltan los dioses del amor, Venus y Cupido, y de las uniones legítimas Juno e Himeneo. Final feliz, pues, para los amores de Maya y Clarisel, los protagonistas de la Tragicomedia que coincide con el final feliz de los amores entre Feliciana y Francisco.

\author{
En los campos Elysios Hymeneo, \\ Juno, y Venus las bodas celebravan \\ De dos esposos, y los coronavan \\ De arrayan, $y$ del árbol de Tymbreo. \\ Caliope, y Euterpe al son que Orfeo, \\ Clio, y Talia en harpas acordavan \\ Sus tálamos felices festejavan \\ De uno, y otro llegados al deseo. \\ De descanso, diziendo, largos años \\ Les dé el divino Amor, y la discordia \\ De sus puertas no passe los umbrales; \\ Entre brocados, y purpúreos paños \\ Maya, y su Clarisel siempre leales \\ Gozen de felicissima concordia. \\ (Pérez 316-317)
}

$\mathrm{Y}$ en esta etapa feliz, Feliciana es Maya, la princesa que consigue el amor de Francisco por su belleza y, lo que es más importante, por su inteligencia ${ }^{19}$. FelicianaMaya no sólo triunfa en el amor, sino que logra la admiración de Sevilla con sus escritos, defiende su preceptiva literaria contra hipotéticos detractores y presume de ser mujer y lograr sus objetivos. Y esta proyección íntima se realiza en el jardín,teatro en el que se funde el mundo real y el ficticio, cómplice de sus sentimientos amorosos y sus anhelos personales, un jardín abierto que simboliza el mundo privado de la autora. La naturaleza domesticada a gusto de su propietaria, donde crece lo que ella quiere y pasa lo que ella quiere que pase.

En conclusión, el motivo del jardín, de una extensísima y compleja tradición literaria, se torna aquí, espacio simbólico ordenado por la mano del artista, en el que se refugia la autora huyendo de una realidad extratextual. Los Jardines y campos Sabeos son, para la autora, un espacio utópico e irreal, un lugar mítico con una reina mítica, un espacio sin puertas ni muros donde a la mujer se le permiten acciones y comportamientos considerados transgresores en el mundo real.

Para Feliciana, entrar en el jardín es entrar en un refugio, escapar a las implacables leyes estéticas y morales de su tiempo. Mientras que en su obra

18 “(...) Quando de amado esposo / El nombre merecido y alcanzado / Te veas, dulce, honroso, / En el solio argentado / De tu bella Diana, y Sol dorado; (...)”./Coro del acto II. (Pérez, 288-289).

${ }^{19}$ Mas la dulçura, mi Maya, / De vuestros versos y rimas, / Es la que ha llegado al alma / Con suave melodía / (II Parte, Acto V, 256, v.342-345). 
paraliteraria (prólogos, dedicatorias, carta a los lectores, carta executoria) se defiende de las acusaciones de los dramaturgos de su época tanto por adoptar los modos clásicos de hacer teatro como por ser mujer en una actividad reservada a varones, en el jardín, su obra es alabada y valorada por hombres y dioses. Mientras que en su vida real deberá someterse a los dictados de una sociedad patriarcal y casarse con un hombre al que no quiere, en el jardín consigue al amor de su vida.

En el jardín, bajo el amparo de la lejanía temporal y el ropaje mitológico dará rienda suelta a sus sentimientos y se expresará con entera libertad. Feliciana convierte así el espacio dramático en un espacio soñado, la interpretación de ese espacio imaginario tiene una funcionalidad clara: es el medio de lograr la autoafirmación de Feliciana como mujer y como autora teatral.

\section{Bibliografía}

A. Egido (ed.) (1989), La escenografía del teatro barroco. Salamanca.

A. Egido (1990), Fronteras de la poesía en el Barroco. Barcelona

C. Hernández Varcárcel (1984), "EI locus amoenus en la Edad Media española" in Simposio Virgiliano. Murcia 321-340.

C. de la Rosa Cubo (2005), "Educación y Herencia Clásica en las escritoras españolas de los siglos XVI y XVII" in Mujeres y educación. Saberes, practicas $y$ discursos en la Historia. Sevilla 161-173.

E. R. Curtius (1977), Literatura europea y Edad Media Latina, Méjico.

J. De Lara Garrido (1995), "El Jardín y la imaginación espacial en el teatro barroco español”, in C. Añón Feliú (Dir.), Jardines y Paisajes en el arte y en la Historia. Madrid 109-156

L. Pérez (1988), The dramatic works of Feliciana Enríquez de Guzmán, Valencia

M. R. Ruiz (2005), Monstruos, mujer y teatro en el barroco. Feliciana Enríquez de Guzmán. Primera dramaturga Española. New York.

O. Arróniz (1977), "Teatros y escenarios del Siglo de Oro”, in J. M. Díez Borque (dir.), (1991), Espacios teatrales del Barroco.Reichenberger. Madrid, Gredos.

P. Conde Parrado, C. de la Rosa (2005) "Una lectura de Ovidio en el drama español del s. XVII: La Tragicomedia de los Jardines y los Campos Sabeos de Feliciana Enríquez de Guzmán”, in Ecos silenciados Burgos. 253-265

T. Ferrer Valls (1991), La práctica escénica cortesana: de la época del Emperador a la de Felipe III. London. 